\title{
Characteristics of coagulation alteration in patients with COVID-19
}

\author{
Hong-chun Luo ${ }^{1} \cdot$ Cheng-yan You ${ }^{2,3,4} \cdot$ Si-wei Lu ${ }^{2,3,4} \cdot$ Yue-qiang Fu ${ }^{2,3,4,5}$ (D)
}

Received: 6 May 2020 / Accepted: 15 October 2020 / Published online: 20 October 2020

(C) Springer-Verlag GmbH Germany, part of Springer Nature 2020

\begin{abstract}
Abnormal blood coagulation often occurs in critically ill patients, which seriously affects their prognosis. This retrospective study investigated the implications of changes in blood coagulation in patients with coronavirus disease 2019 (COVID-19). Records were reviewed for patients admitted with COVID-19 between February 4 and 16, 2020. The primary outcome was in-hospital death. A total of 85 patients were included, of whom 12 died in the hospital. The admission prothrombin time (PT), international normalized ratio (INR), and levels of D-dimer and fibrin/fibrinogen degradation products (FDP) were significantly higher in nonsurvivors than in survivors, while the reverse was true for prothrombin time activity (PT-act) and $\mathrm{PaO}_{2} / \mathrm{FiO}_{2}$. Multivariate logistic regression showed that PT-act $<75 \%$ was independently associated with mortality. The area under the receiver operating characteristic curves for PT-act, D-dimer, and FDP at admission could significantly predict mortality. The AUCs for PT-act were larger than those for D-dimer and FDP; however, there was no significant difference. After 2 weeks of treatment, the coagulation parameters of the surviving patients improved. COVID-19 is often accompanied by abnormal coagulation. PT-act at admission is able to predict mortality in patients with COVID-19 as can D-dimer and FDP levels. PT-act $<75 \%$ is independently associated with mortality.
\end{abstract}

Keywords COVID-19 $\cdot$ Death $\cdot$ Blood coagulation $\cdot$ Prothrombin time activity $\cdot$ D-Dimer

\section{Introduction}

An unknown pneumonia broke out in Wuhan City in December 2019, and it was confirmed as an acute respiratory infectious disease caused by severe acute respiratory syndrome coronavirus 2 (SARS-CoV-2, formerly known as 2019-nCoV). The disease was subsequently named coronavirus disease 2019 (COVID-19) by the WHO on February 11,

Yue-qiang $\mathrm{Fu}$

fuyueqiang@sina.com

1 Department of Infectious Diseases, The First Affiliated Hospital of Chongqing Medical University, Chongqing 400016, People's Republic of China

2 Department of Critical Care Medicine, Children's Hospital, Chongqing Medical University, 136\# Zhongshan Er Road, Yu Zhong District, Chongqing 400014, People's Republic of China

3 Ministry of Education Key Laboratory of Child Development and Disorders, Chongqing 400014, People's Republic of China

4 National Clinical Research Center for Child Health and Disorders (Chongqing), Chongqing 400014, People's Republic of China

5 Third Batch Chongqing Medical Aid Team to Wuhan City of Hubei Province, Chongqing, People's Republic of China
2020. SARS-CoV-2 is highly contagious and can cause serious lung injury, resulting in death, and COVID-19 has been widely spreading and causing great damage in many countries around the world.

Pneumonia, especially severe pneumonia, can lead to abnormal coagulation $[1,2]$. Recently, some studies reported that severe COVID-19 is commonly complicated with coagulopathy, and elevated levels of D-dimer and fibrin/fibrinogen degradation products (FDP) were associated with poor prognosis in severe COVID-19 [3-6]. SARS-CoV-2 may activate the innate immune system to clear the virus; however, excessive immune responses can cause inflammatory storms, damage microcirculation, activate the blood coagulation system, and lead to disseminated intravascular coagulation (DIC).

The influence of coagulation dysfunction on the prognosis of COVID-19 is attracting increasing attention [3]. However, studies on the predictive and prognostic values of coagulation parameters in the setting of patients with COVID-19 are still limited. The purpose of this retrospective case-control study was to investigate the predictive values of coagulation parameters for in-hospital mortality in patients with COVID-19 and to compare them. In addition, independent risk factors for mortality were also explored. 


\section{Patients and methods}

\section{Study design}

We performed a retrospective cohort study of patients with COVID-19 who were admitted to the wards of the Third Batch of Chongqing Medical Aid Team in Wuhan City of Hubei Province in China from February 4, 2020, to February 16, 2020.

The Ethics Committee of Children's Hospital, Chongqing Medical University (Institutional Review Board of Children's Hospital, Chongqing Medical University), approved this retrospective cohort study. The patient records were anonymized prior to the analysis.

From February 4, 2020, to February 16, 2020, consecutive patients in the wards with SARS-CoV-2 infection confirmed by real-time reverse transcription polymerase chain reaction (RT-PCR) assay from nose and throat swab samples, and with pneumonia confirmed by computed tomography (CT) examination and coagulation test completed within $12 \mathrm{~h}$ after admission were enrolled. Patients with specific cardiovascular disease who used anticoagulants before admission were also excluded.

Coagulation tests, which included prothrombin time (PT), prothrombin time activity (PT-act), the international normalized ratio (INR), activated partial thromboplastin time (APTT), thrombin time (TT), fibrinogen (Fib), D-dimer, fibrin/FDP, and antithrombin III activity, were performed using a Sysmex CS5100 automatic coagulation analyzer (Japan) and proprietary reagents.

\section{Data collection}

The variables examined were age, sex, body mass index (BMI), comorbidities, PT, PT-act, INR, APTT, TT, Fib, Ddimer, FDP, antithrombin III activity, $\mathrm{PaO}_{2} / \mathrm{FiO}_{2}$, lymphocyte count, platelet (PLT) count, chest computer tomography, and the application of prophylactic anticoagulation after admission.

\section{Outcome}

The primary outcome was in-hospital death.

\section{Statistical analysis}

Data were analyzed using SPSS 21.0 (SPSS Inc. Chicago, IL, USA). Continuous variables are shown as medians (interquartile ranges, IQRs), and categorical data are shown as percentages. Comparisons of two medians were performed using the Mann-Whitney $U$ test, and comparisons of percentages were performed with the chi-square test. Multivariate analysis was performed to determine whether PT-act $<75 \%$ was a risk factor for mortality after adjusting for other variables. Receiver operating characteristic (ROC) curves (MedCalc v15.11.4, Ostend, Belgium) were used to determine blood coagulation parameters at admission as predictors of mortality. The median coagulation parameters at admission and 2 weeks later were compared using the Wilcoxon test. A $P$ value $<0.05$ was considered statistically significant for all analyses.

\section{Results}

\section{Clinical characteristics}

A total of 93 patients were admitted during the study period, 85 of whom met the inclusion criteria for this study. Eight patients were excluded according to the exclusion criteria. The median age of the patients was $63.0(54.5,70.0)$ years, ranging from 31 to 89 years. Forty-eight $(48 / 85,56.47 \%)$ patients were male, and $45(45 / 85,52.94 \%)$ patients had at least one chronic comorbidity. Hypertension (35.29\%), diabetes $(14.12 \%)$, and coronary heart disease $(11.76 \%)$ were the most common comorbidities. The percentages of admission $\mathrm{PaO}_{2} / \mathrm{FiO}_{2}<200$ and $\mathrm{CRP}>80 \mathrm{mg} / \mathrm{L}$ in the non-survivors were significantly higher than those in the survivors. Compared with those of survivors, the lymphocyte and platelet counts of non-survivors were significantly lower. Prophylactic anticoagulant therapy (enoxaparin, $40 \mathrm{mg}$ once daily) was used in 47 patients for approximately 5 days after admission. Comparisons of the demographic and clinical characteristics of the survivors and non-survivors are shown in Table 1.

\section{Coagulation parameters of survivors and non- survivors}

The admission PT, INR, and levels of D-dimer and FDP were significantly higher in the non-survivors than in the survivors, while the reverse was true for PT-act (Table 2). Meanwhile, the percentages of those with PT $>13 \mathrm{~s}$, PT-act $<75 \%$, Ddimer $>0.55 \mathrm{mg} / \mathrm{L}$, and FDP $>5 \mathrm{mg} / \mathrm{L}$ were significantly higher in non-survivors than in survivors (Table 2).

Univariate and multivariate logistic regression analyses were performed to determine whether admission PT-act < $75 \%$ was independently associated with mortality (Table 3 ). Admission PT-act $<75 \%$ (adjusted odds ratio $(\mathrm{OR})=25.623$; 95\% confidence interval (CI): $2.639-248.750 ; P=0.005$ ) was an independent risk factor for mortality when the other variables were controlled.

\section{ROC curve analyses of mortality}

Area under the curve (AUC) analysis was used to predict mortality. Admission PT-act D-dimer and FDP could predict 
Table 1 Demographics and clinical characteristics of the survivors and non-survivors

\begin{tabular}{lllll}
\hline & All patients & Survivors & Non-survivors & $P$ \\
\hline Subjects, $n$ & 85 & 73 & 12 & - \\
Age (years) & $63.00(54.50,70.00)$ & $62.00(54.5,69.50)$ & $67.00(52.50,74.75)$ & 0.340 \\
Gender, male, $n(\%)$ & $48(56.47 \%)$ & $39(53.42 \%)$ & $9(75.00 \%)$ & 0.162 \\
BMI & $23.14(21.70,24.62)$ & $23.14(21.67,24.23)$ & $23.63(21.99,27.03)$ & 0.281 \\
Any comorbidity, $n(\%)$ & $45(52.94 \%)$ & $36(49.32 \%)$ & $9(75.00 \%)$ & 0.099 \\
Hypertension, $n(\%)$ & $30(35.29 \%)$ & $24(32.88 \%)$ & $6(50.00 \%)$ & 0.250 \\
Coronary heart disease, $n$ & $10(11.76 \%)$ & $8(10.96 \%)$ & $2(16.67 \%)$ & 0.570 \\
$\quad(\%)$ & $12(14.12 \%)$ & $9(12.33 \%)$ & $3(25.00 \%)$ & 0.243 \\
Diabetes, $n(\%)$ & $5(5.88 \%)$ & $3(4.11 \%)$ & $2(16.77 \%)$ & 0.087 \\
COPD, $n(\%)$ & $13.00(10.00,15.00)$ & $13.00(10.00,15.50)$ & $12.00(9.25,14.75)$ & 0.429 \\
Illness onset to admission & & & \\
$\quad$ days $)$ & $0.97(0.64,1.42)$ & $1.00(0.77,1.43)$ & $0.59(0.51,0.85)$ & 0.009 \\
Lymphocyte counts $(\times$ & $218.00(164.50$, & $222.00(181.50$, & $161.00(126.25$, & 0.013 \\
$\left.\quad 10^{9} / \mathrm{L}\right)$ & $270.00)$ & $274.00)$ & $238.75)$ & 0.006 \\
PLT & $22(25.88 \%)$ & $15(20.55 \%)$ & $7(58.33 \%)$ & 0.004 \\
CRP $>80$ mg/L, $n(\%)$ & $32(37.65 \%)$ & $23(31.51 \%)$ & $9(75.00 \%)$ & 0.819 \\
PaO $/$ FiO $2<200, n(\%)$ & $47(55.29 \%)$ & $40(54.79 \%)$ & $7(58.33 \%)$ & \\
Prophylactic & & & & \\
$\quad$ anticoagulation & & & & \\
\hline
\end{tabular}

$B M I$ body mass index, $C O P D$ chronic obstructive pulmonary disease, $C R P$ C-reactive protein, $P L T$ platelet mortality in patients with COVID-19 (Table 4 and Fig. 1). The AUC for PT-act was 0.905 (95\% CI: 0.822 to $0.958, P<$ 0.001 ), that for D-dimer was 0.816 (95\% CI: 0.717 to 0.891 , $P<0.001)$, and that for FDP was 0.830 (95\% CI: 0.734 to $0.903, P<0.001$ ) (Table 4). The AUCs for admission PT-act were larger than those for admission D-dimer and FDP in patients with COVID-19; however, there were no statistically significant differences (AUC for PT-act vs. AUC for D-dimer, $P=0.216$; AUC for PT-act vs. AUC for FDP, $P=0.281)$. The cut-off point of $76 \%$ that was obtained for the PT-act area had the maximum sensitivity (100\%) and specificity $(78.08 \%)$ for predicting mortality (Table 4).

\section{Dynamic profile of coagulation parameters after 2 weeks of hospitalization in survivors}

In the survival group ( $n=73$ ), the condition of patients improved, and chest CT also gradually improved. Four patients were well enough for discharge within 2 weeks after admission. Fifty-eight hospitalized survivors underwent coagulation tests 2 weeks after treatment. After 2 weeks of hospitalization, PT, INR, APTT, and FIB were significantly lower than at baseline, while the reverse was true for PT-act (Table 5). Furthermore, among 12 patients whose admission PT-act was < $75 \%, 10$ patients had PT-act values that became higher than $75 \%$ after 2 weeks of treatment (Table 5 ).

Finally, all survivors $(n=73)$ were discharged.

\section{Discussion}

Coagulation dysfunction seems to be an important issue in patients with COVID-19. Recently, some researchers analyzed the clinical and laboratory findings of COVID-19 and found that severe patients often had prolonged PT, increased D-dimer levels, low fibrinogen, and DIC [6, 7]. Inflammatory storms are a feature of severe COVID-19. Compared with moderate patients, severe patients more frequently had dyspnea and lymphopenia, with markedly higher levels of IL-2R, IL-6, IL-10, and TNF- $\alpha$ [8]. The severe inflammatory state secondary to COVID-19 leads to severe derangement of hemostasis and prominent alternation of coagulation parameters $[4,9-11]$. It is generally believed that deterioration of coagulation parameters during the disease course is closely associated with COVID-19 worsening and death.

As one of the most important parameters, PT is widely used to assess coagulation function in the clinic. Many studies have explored the change in PT in patients with COVID-19 [7-9, 12-15]. Chen et al. [8] showed that the PT was not significantly different between severe cases and moderate cases with COVID-19. Han et al. [5] also reported that there was no significant difference in PT between patients with different levels of severity of COVID-19 and healthy controls. However, more studies reported that severe patients had significantly prolonged PT compared with non-severe patients [16-18]. In addition, two studies on COVID-19 indicated that non-survivors had higher PT levels than survivors [6, 12]. We also found that admission PT was significantly higher in non- 
Table 2 Comparison of coagulation parameters between survivors and non-survivors

\begin{tabular}{|c|c|c|c|c|c|}
\hline & Normal range & All patients & Survivors & Non-survivors & $P$ \\
\hline Subject & - & 85 & 73 & 12 & - \\
\hline $\mathrm{PT}(\mathrm{s})$ & $9-13$ & $12.20(11.55,12.75)$ & $12.00(11.50,12.50)$ & $13.05(12.80,14.00)$ & 0.000 \\
\hline $9-13, n(\%)$ & & $70(82.35 \%)$ & $64(87.67 \%)$ & $6(50 \%)$ & 0.002 \\
\hline$>13, n(\%)$ & & $15(17.65 \%)$ & $9(12.33 \%)$ & $6(50 \%)$ & 0.002 \\
\hline PT-act (\%) & $75-135$ & $81.70(74.83,92.75)$ & $84.70(77.40,93.28)$ & $71.2(62.45,74.80)$ & 0.000 \\
\hline$<75, n(\%)$ & & $27(31.76 \%)$ & $16(21.92 \%)$ & $11(91.67 \%)$ & 0.000 \\
\hline $75-135, n(\%)$ & & $58(68.24 \%)$ & $57(78.08 \%)$ & $1(8.33 \%)$ & \\
\hline INR & $0.76-1.24$ & $1.05(0.99,1.10)$ & $1.03(0.98,1.07)$ & $1.13(1.10,1.21)$ & 0.000 \\
\hline $0.76-1.24, n(\%)$ & & $83(97.65 \%)$ & $73(100 \%)$ & $10(83.33 \%)$ & 0.000 \\
\hline$>1.24, n(\%)$ & & $2(2.35 \%)$ & 0 & $2(16.67 \%)$ & \\
\hline APTT (s) & $25-31.3$ & $29.40(26.60,31.85)$ & $29.50(26.30,31.65)$ & $29.30(27.78,32.80)$ & 0.319 \\
\hline$<25, n(\%)$ & & $6(7.06 \%)$ & $6(8.22 \%)$ & 0 & 0.488 \\
\hline $25-31.3, n(\%)$ & & $52(61.18 \%)$ & $45(61.64 \%)$ & $7(58.33 \%)$ & \\
\hline$>31.3, n(\%)$ & & $27(31.76 \%)$ & $22(30.14 \%)$ & $5(41.67 \%)$ & \\
\hline TT (s) & $14-21$ & $18.40(17.20,19.70)$ & $18.00(17.20,19.70)$ & $19.35(18.03,22.60)$ & 0.079 \\
\hline $14-21, n(\%)$ & & $77(90.59 \%)$ & $69(94.52 \%)$ & $8(66.67 \%)$ & 0.002 \\
\hline$>21, n(\%)$ & & $8(9.41 \%)$ & $4(5.48 \%)$ & $4(33.33 \%)$ & \\
\hline Fib $(g / L)$ & $2-4$ & $4.56(3.33,5.24)$ & $4.56(3.48,5.26)$ & $3.62(2.48,5.30)$ & 0.218 \\
\hline$<2, n(\%)$ & & $4(4.71 \%)$ & $2(2.74 \%)$ & $2(16.67 \%)$ & 0.025 \\
\hline $2-4, n(\%)$ & & $28(32.94 \%)$ & $22(30.14 \%)$ & $6(50.00 \%)$ & \\
\hline$>4, n(\%)$ & & $53(62.35 \%)$ & $49(67.12 \%)$ & $4(33.33 \%)$ & \\
\hline D-Dimer (mg/L) & $0-0.55$ & $0.76(0.39,2.21)$ & $0.63(0.34,1.46)$ & $4.11(0.81,52.69)$ & 0.000 \\
\hline $0-0.55, n(\%)$ & & $35(41.18 \%)$ & $34(46.58 \%)$ & $1(8.33 \%)$ & 0.013 \\
\hline$>0.55, n(\%)$ & & $50(58.82 \%)$ & $39(53.42 \%)$ & $11(91.67 \%)$ & \\
\hline $\mathrm{FDP}(\mathrm{mg} / \mathrm{L})$ & $0-5$ & $2.82(1.09,7.60)$ & $1.93(0.94,5.14)$ & $31.00(3.61,108.86)$ & 0.000 \\
\hline $0-5, n(\%)$ & & $59(69.41 \%)$ & $55(75.34 \%)$ & $4(33.33 \%)$ & 0.003 \\
\hline$>5, n(\%)$ & & $26(30.59 \%)$ & $18(24.66 \%)$ & $8(66.67 \%)$ & \\
\hline Antithrombin III activity (\%) & $80-120$ & $86.00(78.20,94.15)$ & $86.80(78.50,94.70)$ & $80.10(70.95,89.30)$ & 0.100 \\
\hline$<80, n(\%)$ & & $25(29.41 \%)$ & $19(26.03 \%)$ & $6(50 \%)$ & 0.091 \\
\hline $80-120, n(\%)$ & & $60(70.59 \%)$ & $54(73.97 \%)$ & $6(50 \%)$ & \\
\hline
\end{tabular}

APTT activated partial thromboplastin time, $F D P$ fibrin/fibrinogen degradation products, Fib fibrinogen, INR international normalized ratio, $P T$ prothrombin time, $P T$-act prothrombin time activity, $T T$ thrombin time

Table 3 Univariate and multivariate logistic regression to identify risk factors at admission related to mortality

\begin{tabular}{|c|c|c|c|c|}
\hline & $\begin{array}{l}\text { Univariate } \\
\text { Unadjusted OR }(95 \% \mathrm{CI})\end{array}$ & $P$ & $\begin{array}{l}\text { Multivariate } \\
\text { Adjusted OR }(95 \% \mathrm{CI})\end{array}$ & $P$ \\
\hline PT-act $<75 \%$ & $42.533(5.083-355.908)$ & 0.001 & $25.623(2.639-248.750)$ & 0.005 \\
\hline $\mathrm{D}$-dimer $>0.55 \mathrm{mg} / \mathrm{L}$ & $9.075(1.113-73.987)$ & 0.039 & $2.146(0.146-31.547)$ & 0.578 \\
\hline $\mathrm{FDP}>5 \mathrm{mg} / \mathrm{L}$ & $6.111(1.644-22.715)$ & 0.007 & $2.623(0.387-17.762)$ & 0.323 \\
\hline $\mathrm{Fib}<2.00 \mathrm{~g} / \mathrm{L}$ & $7.100(0.897-56.196)$ & 0.063 & $99.886(0.133-74878.420)$ & 0.173 \\
\hline $\mathrm{PaO}_{2} / \mathrm{FiO}_{2}<200$ & $6.522(1.613-26.364)$ & 0.009 & $3.082(0.549-17.310)$ & 0.201 \\
\hline Lymphocyte counts $\left(\times 10^{9} / \mathrm{L}\right)$ & $0.277(0.059-1.289)$ & 0.102 & $0.219(0.011-4.433)$ & 0.323 \\
\hline
\end{tabular}

$C I$ confidence interval, $F D P$ fibrin/fibrinogen degradation products, $O R$ odds ratio, $P T$-act prothrombin time activity 
Table 4 AUCs determined from ROC curves for predictors of mortality due to COVID-19

\begin{tabular}{lllllll}
\hline & AUC & $95 \% \mathrm{CI}$ & $P$ & Optimal cut-off value & Sensitivity & Specificity \\
\hline PT-act & 0.905 & $0.822 \sim 0.958$ & $<0.001$ & $76 \%$ & 100.00 & 78.08 \\
D-Dimer & 0.816 & $0.717 \sim 0.891$ & $<0.001$ & $2.52 \mathrm{mg} / \mathrm{L}$ & 66.67 & 83.56 \\
FDP & 0.830 & $0.734 \sim 0.903$ & $<0.001$ & $9.66 \mathrm{mg} / \mathrm{L}$ & 66.67 & 86.30 \\
\hline
\end{tabular}

$A U C$ area under the curve, $C I$ confidence interval, FDP fibrin/fibrinogen degradation products, $P T$-act prothrombin time activity survivors than in survivors, while the admission PT values of most patients $(70 / 85,82.35 \%)$ were in the normal range ( 9 to $13 \mathrm{~s}$ ) in the present study.

It is worth noting that variability in thromboplastin reagents may lead to large interlaboratory differences in PT results. The INR is widely used in the clinic and is able to make the results of different laboratories comparable by standardizing different reagents: $\mathrm{INR}=\left(\mathrm{PT}_{\text {test }} / \mathrm{PT}_{\text {normal }}\right)^{\mathrm{ISI}}$, where $\mathrm{PT}_{\text {test }}$ refers to the $\mathrm{PT}$ value of patient, $\mathrm{PT}_{\text {normal }}$ refers to the $\mathrm{PT}$ value of normal control health, and ISI indicates the International Sensitivity Index of the thromboplastin reagent used. In the present study, we found that the INR values were significantly higher in nonsurvivors than in survivors; however, $97.65 \%$ of INR values at admission were within the normal range (0.76-1.24).

PT-act also provides a common international scale for PT reporting as a supplement. The calculation formula of PT-act is as follows: $\mathrm{PT}$-act $=\left[\mathrm{PT}_{\text {normal }}-\left(\mathrm{PT}_{\text {normal }} \times 0.6\right)\right] /\left[\mathrm{PT}_{\text {test }}-\right.$ $\left.\left(\mathrm{PT}_{\text {normal }} \times 0.6\right)\right] \times 100 \%$. PT-act has the similar clinical significance as PT and can accurately reflect the activity of coagulation factors II, V, VII, and X. The decrease in coagulation factor synthesis, DIC, and hyperfibrinolysis could

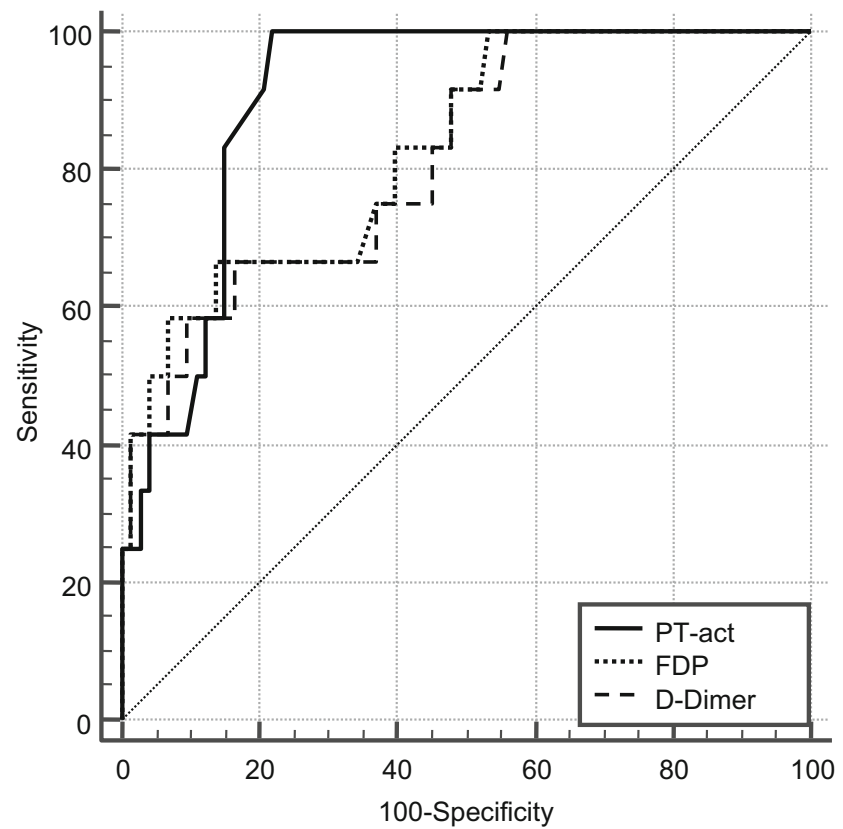

Fig. 1 Receiver operating characteristic curves for the ability of D-Dimer, FDP, and PT-act to predict in-hospital mortality in patients with COVID19 significantly prolong PT and decrease PT-act. In this study, PT-act was significantly lower in non-survivors than in survivors. We also show that the incidence of PT-act $<75 \%$ at admission was markedly high $(91.67 \%)$ in non-survivors, while it was $21.92 \%$ in survivors, and the percentage of abnormal PT and INR values in non-survivors was $50 \%$ and $16.67 \%$, respectively. Therefore, we argue that PT-act may better reflect the severity of patients than PT and the INR.

At present, there is only one study involving PT-act in patients with COVID-19 [5]. Han et al. [5] reported that PTact was lower in SARS-CoV-2 patients compared to healthy controls and that PT-act decreased with increasing severity of the disease; however, this study did not explore the effect of PT-act on the outcome of patients with COVID-19. The researchers did not focus on the PT-act, which may be because the PT-act was not a conventional indicator of coagulation function in the clinic, and some hospitals did not carry out PT-act testing.

Many studies on COVID-19 have focused on the D-dimer level. COVID-19 patients with D-dimer levels $\geq 2.0 \mu \mathrm{g} / \mathrm{mL}$ had a higher incidence of mortality than those with D-dimer levels $<2.0 \mu \mathrm{g} / \mathrm{mL}$, and the authors argued that D-dimer could be an early and helpful marker to improve the management of COVID-19 patients [19]. Another study showed that 16 patients with COVID-19 ARDS had elevated D-dimer levels (5.5 $\mu \mathrm{g} / \mathrm{mL}$, interquartile range 2.5-6.5) [20]. A metaanalysis showed that approximately $37.2 \%$ of patients with COVID-19 had an elevated D-dimer level [21]. The significant elevation of D-dimer in severe novel coronavirus pneumonia (NCP) patients was a good index for identifying groups at high risk of venous thromboembolism [13]. Tang et al. [6] found that markedly elevated D-dimer and FDP levels are common in deaths due to NCP. Tang et al. [14] used heparin to treat patients and reported that the 28-day mortality of heparin users was lower than that of non-users in patients with Ddimer levels $>6$-fold of the upper limit of normal (32.8\% vs. $52.4 \%, P=0.017)$. Our study showed that the levels of $\mathrm{D}$ dimer in $58.82 \%(50 / 85)$ of patients with COVID-19 were larger than the upper limit of normal $(0.55 \mathrm{mg} / \mathrm{L})$, and the non-survivors had significantly higher D-dimer levels than the survivors.

Multivariate logistic regression analysis showed that admission PT-act $<75 \%$ was an independent risk factor for 
Table 5 Coagulation parameters in the survival group at admission and after 2 weeks of admission

\begin{tabular}{|c|c|c|c|c|}
\hline & Normal range & Admission & After 2 weeks & $P$ \\
\hline Subject & & 58 & 58 & \\
\hline PT (s) & $9-13$ & $12.00(11.50,12.53)$ & $11.20(10.70,11.53)$ & 0.000 \\
\hline $9-13, n(\%)$ & & $50(86.21 \%)$ & $56(96.55 \%)$ & 0.047 \\
\hline$>13, n(\%)$ & & $8(13.79 \%)$ & $2(3.45 \%)$ & \\
\hline PT-act (\%) & $75-135$ & $84.70(76.98,93.23)$ & $99.80(92.80,107.48)$ & 0.000 \\
\hline$<75, n(\%)$ & & $12(20.69 \%)$ & $2(3.45 \%)$ & 0.011 \\
\hline $75-135, n(\%)$ & & $46(79.31 \%)$ & $55(94.83 \%)$ & \\
\hline$>135, n(\%)$ & & 0 & $1(1.72 \%)$ & \\
\hline INR & $0.76-1.24$ & $1.03(0.98,1.07)$ & $0.95(0.92,0.98)$ & 0.000 \\
\hline $0.76-1.24$ & & $58(100 \%)$ & $58(100 \%)$ & \\
\hline APTT (s) & $25-31.3$ & $29.60(26.30,31.60)$ & $26.20(25.28,27.50)$ & 0.000 \\
\hline$<25, n(\%)$ & & $3(3.53 \%)$ & $13(22.41 \%)$ & 0.000 \\
\hline $25-31.3, n(\%)$ & & $38(65.52 \%)$ & $43(74.14 \%)$ & \\
\hline$>31.3, n(\%)$ & & $17(29.31 \%)$ & $2(3.45 \%)$ & \\
\hline TT (s) & $14-21$ & $18.30(17.20,19.70)$ & $18.10(17.40,19.03)$ & 0.533 \\
\hline $14-21, n(\%)$ & & $54(93.10 \%)$ & $58(100 \%)$ & 0.042 \\
\hline$>21, n(\%)$ & & $4(6.90 \%)$ & 0 & \\
\hline $\mathrm{FIB}(\mathrm{g} / \mathrm{L})$ & $2-4$ & $4.50(3.37,5.12)$ & $2.96(2.52,3.41)$ & 0.000 \\
\hline$<2$ & & $1(1.72 \%)$ & $4(6.90 \%)$ & 0.000 \\
\hline $2-4$ & & $20(34.48 \%)$ & $47(81.03 \%)$ & \\
\hline$>4$ & & $37(63.79 \%)$ & $7(12.07 \%)$ & \\
\hline D-Dimer (mg/L) & $0-0.55$ & $0.77(0.38,1.71)$ & $0.59(0.38,0.98)$ & 0.058 \\
\hline $0-0.55, n(\%)$ & & $25(43.10 \%)$ & $28(48.28 \%)$ & 0.576 \\
\hline$>0.55, n(\%)$ & & $33(56.90 \%)$ & $30(51.72 \%)$ & \\
\hline $\mathrm{FDP}(\mathrm{mg} / \mathrm{L})$ & $0-5$ & $1.93(0.93,5.67)$ & $1.84(0.56,4.75)$ & 0.146 \\
\hline $0-5, n(\%)$ & & $42(72.41 \%)$ & $45(77.59 \%)$ & 0.520 \\
\hline$>5, n(\%)$ & & $16(27.59 \%)$ & $13(22.41 \%)$ & \\
\hline Antithrombin III activity (\%) & $80-120$ & $87.80(78.20,95.38)$ & $90.50(80.38,95.88)$ & 0.126 \\
\hline$<80, n(\%)$ & & $16(27.59 \%)$ & $13(22.41 \%)$ & 0.520 \\
\hline $80-120, n(\%)$ & & $42(72.41 \%)$ & $45(77.59 \%)$ & \\
\hline
\end{tabular}

$A P T T$ activated partial thromboplastin time, $F D P$ fibrin/fibrinogen degradation products, FIB fibrinogen, INR international normalized ratio, $P T$ prothrombin time, $P T$-act prothrombin time activity, $T T$ thrombin time mortality in patients with COVID-19 in the present study; however, admission D-dimer $>0.55 \mathrm{mg} / \mathrm{L}$ was not an independent risk factor for mortality. Zhou et al. [12] reported that multivariable regression showed increasing odds of inhospital death associated with D-dimer $>1 \mu \mathrm{g} / \mathrm{mL}$ on admission. Liu et al. [22] found that increased D-dimer levels at admission were closely related to death through multivariable logistic regression. Wu et al. [23] also reported that D-dimer was associated with progression from ARDS to death in bivariate Cox regression analysis. Of course, the differences between our study and other studies may be due to the different research designs and indicator screening. However, the value of PT-act at admission should be given adequate attention when treating patients with COVID-19.

The accuracy of coagulation parameters for predicting inhospital mortality was evaluated using ROC curve analysis.
We found that PT-act, D-dimer, and FDP are significant predictors of mortality. Combined with the fact that PT-act $<75 \%$ is an independent risk factor for patient death, we argue that PT-act is a useful index for predicting COVID-19 patient death. Liu et al. [11] reported that using ROC analyses, the AUC values for PT, FDP, and D-dimer at admission were $0.892,0.81$, and 0.809 , respectively, for predicting inhospital mortality in patients with COVID-19. Another study on COVID-19 also indicated that the AUCs of PT and Ddimer at admission were 0.643 and 0.742 for predicting mortality, and they increased to 0.937 and 0.851 , respectively, at the composite endpoint [22]. Combined with these studies, we argue that the specific parameters of coagulation function, including PT-act at admission, can effectively predict the prognosis of patients with COVID-19. Although serial measurements may provide more information and guide 
treatment, testing coagulation parameters at admission still has the advantage of providing doctors with key information in a timely manner and helping doctors give relevant treatments quickly at the early stage of hospitalization.

This study has several limitations. First, this retrospective study was limited by factors that are inherent to retrospective analysis. Second, this was a single-center retrospective study, and the results may not be representative due to the small sample size. However, it may be the first clinical study concerning the predictive value of PT-act at admission for mortality in patients with COVID-19. A multicenter study with a larger sample size is needed to verify our results. Third, we only collected blood coagulation tests at admission and 2 weeks after hospitalization, which may not accurately reflect the continuous dynamic changes of coagulation. Fourth, because there is no ultrasound screening of blood vessels, we do not have data about the occurrence of thrombi in patients. Last, the relatively high mortality rate is related to the severe condition of the patients (the admission $\mathrm{PaO}_{2} / \mathrm{FiO}_{2}$ of 68 patients was less than 300) and inadequate early medical conditions. Despite these limitations, we found that PT-act at admission has a predictive and prognostic value, which can enable clinicians to identify patients with COVID-19 who are at a great risk of death in the early stage of the disease.

Finally, it was needed to point out that 81 of the 85 patients included in the present study were also part of other study [24], which did not involve coagulation.

\section{Conclusions}

COVID-19 is often accompanied by abnormal coagulation. PT-act at admission is able to predict mortality in patients with COVID-19 as can D-dimer and FDP levels. Moreover, PT-act $<75 \%$ is independently associated with mortality.

Authors' contribution YF participated in the design, data acquisition, database management, statistical analysis, and coordination. HL participated in the statistical analysis and drafted the manuscript. CY and SL participated in the database management and statistical analysis. YF has responsibility for the article as a whole.

Funding The clinical research project of COVID-19 in Chongqing Medical University (Grant No. 0064)

Data availability All relevant data are within the paper.

\section{Compliance with ethical standards}

The study was conducted in accordance with the Declaration of Helsinki, the protocol was approved by the Institutional Review Board of Children's Hospital, Chongqing Medical University, and the requirement for written informed consent was waived because of the retrospective design.
Competing interests The authors declare that they have no competing interests.

\section{References}

1. Idell S (2003) Coagulation, fibrinolysis, and fibrin deposition in acute lung injury. Crit Care Med 31(4 Suppl):S213-S220. https:// doi.org/10.1097/01.CCM.0000057846.21303.AB

2. Welty-Wolf KE, Carraway MS, Ortel TL, Piantadosi CA (2002) Coagulation and inflammation in acute lung injury. Thromb Haemost 88(1):17-25

3. Terpos E, Ntanasis-Stathopoulos I, Elalamy I, Kastritis E, Sergentanis TN, Politou M, Psaltopoulou T, Gerotziafas G, Dimopoulos MA (2020) Hematological findings and complications of COVID-19. Am J Hematol 95:834-847. https://doi.org/10.1002/ ajh.25829

4. Panigada M, Bottino N, Tagliabue P, Grasselli G, Novembrino C, Chantarangkul V, Pesenti A, Peyvandi F, Tripodi A (2020) Hypercoagulability of COVID-19 patients in intensive care unit. A report of thromboelastography findings and other parameters of hemostasis. J Thromb Haemost 18:1738-1742. https://doi.org/10. 1111 /jth. 14850

5. Han H, Yang L, Liu R, Liu F, Wu KL, Li J, Liu XH, Zhu CL (2020) Prominent changes in blood coagulation of patients with SARSCoV-2 infection. Clin Chem Lab Med 58:1116-1120. https://doi. org $/ 10.1515 / \mathrm{cclm}-2020-0188$

6. Tang N, Li D, Wang X, Sun Z (2020) Abnormal coagulation parameters are associated with poor prognosis in patients with novel coronavirus pneumonia. J Thromb Haemost 18(4):844-847. https:// doi.org/10.1111/jth.14768

7. Giannis D, Ziogas IA, Gianni P (2020) Coagulation disorders in coronavirus infected patients: COVID-19, SARS-CoV-1, MERS$\mathrm{CoV}$ and lessons from the past. J Clin Virol 127:104362. https://doi. org/10.1016/j.jcv.2020.104362

8. Chen G, Wu D, Guo W, Cao Y, Huang D, Wang H, Wang T, Zhang X, Chen $\mathrm{H}$, Yu H, Zhang X, Zhang M, Wu S, Song J, Chen T, Han M, Li S, Luo X, Zhao J, Ning Q (2020) Clinical and immunologic features in severe and moderate coronavirus disease 2019. J Clin Invest 130(5):2620-2629. https://doi.org/10.1172/ JCI137244

9. Chauhan AJ, Wiffen LJ, Brown TP (2020) COVID-19: a collision of complement, coagulation and inflammatory pathways. J Thromb Haemost. https://doi.org/10.1111/jth.14981

10. Fei Y, Tang N, Liu H, Cao W (2020) Coagulation dysfunction: a hallmark in COVID-19. Arch Pathol Lab Med 144:1223-1229. https://doi.org/10.5858/arpa.2020-0324-SA

11. Liu Y, Gao W, Guo W, Guo Y, Shi M, Dong G, Ge Q, Zhu J, Lu J (2020) Prominent coagulation disorder is closely related to inflammatory response and could be as a prognostic indicator for ICU patients with COVID-19. J Thromb Thrombolysis. https://doi.org/ 10.1007/s11239-020-02174-9

12. Zhou F, Yu T, Du R, Fan G, Liu Y, Liu Z, Xiang J, Wang Y, Song B, Gu X, Guan L, Wei Y, Li H, Wu X, Xu J, Tu S, Zhang Y, Chen H, Cao B (2020) Clinical course and risk factors for mortality of adult inpatients with COVID-19 in Wuhan, China: a retrospective cohort study. Lancet 395(10229):1054-1062. https://doi.org/10. 1016/S0140-6736(20)30566-3. Erratum in: Lancet 395(10229): 1038. https://doi.org/10.1016/S0140-6736(20)30606-1. Erratum in: Lancet 395(10229):1038. https://doi.org/10.1016/S01406736(20)30638-3

13. Cui S, Chen S, Li X, Liu S, Wang F (2020) Prevalence of venous thromboembolism in patients with severe novel coronavirus pneumonia. J Thromb Haemost 18(6):1421-1424. https://doi.org/10. $1111 /$ jth. 14830 
14. Tang N, Bai H, Chen X, Gong J, Li D, Sun Z (2020) Anticoagulant treatment is associated with decreased mortality in severe coronavirus disease 2019 patients with coagulopathy. J Thromb Haemost 18(5):1094-1099. https://doi.org/10.1111/jth.14817

15. Liu Q, Song NC, Zheng ZK, Li JS, Li SK (2020) Laboratory findings and a combined multifactorial approach to predict death in critically ill patients with COVID-19: a retrospective study. Epidemiol Infect 148:e129. https://doi.org/10.1017/ S0950268820001442

16. Jin S, Jin Y, Xu B, Hong J, Yang X (2020) Prevalence and impact of coagulation dysfunction in COVID-19 in China: a meta-analysis [published online ahead of print, 2020 Jul 17]. Thromb Haemost. https://doi.org/10.1055/s-0040-1714369

17. Liao D, Zhou F, Luo L, Xu M, Wang H, Xia J, Gao Y, Cai L, Wang Z, Yin P, Wang Y, Tang L, Deng J, Mei H, Hu Y (2020) Haematological characteristics and risk factors in the classification and prognosis evaluation of COVID-19: a retrospective cohort study. Lancet Haematol S2352-3026(20):30217-30219. https:// doi.org/10.1016/S2352-3026(20)30217-9

18. Bao C, Tao X, Cui W, Yi B, Pan T, Young KH, Qian W (2020) SARS-CoV-2 induced thrombocytopenia as an important biomarker significantly correlated with abnormal coagulation function, increased intravascular blood clot risk and mortality in COVID-19 patients. Exp Hematol Oncol 9:16. https://doi.org/10.1186/s40164020-00172-4

19. Zhang L, Yan X, Fan Q, Liu H, Liu X, Liu Z, Zhang Z (2020) Ddimer levels on admission to predict in-hospital mortality in patients with Covid-19. J Thromb Haemost 18(6):1324-1329. https://doi. org/10.1111/jth.14859
20. Ranucci M, Ballotta A, Di Dedda U, Bayshnikova E, Dei Poli M, Resta M, Falco M, Albano G, Menicanti L (2020) The procoagulant pattern of patients with COVID-19 acute respiratory distress syndrome. J Thromb Haemost 18(7):1747-1751. https://doi.org/10. 1111/jth.14854

21. Zhu J, Ji P, Pang J, Zhong Z, Li H, He C, Zhang J, Zhao C (2020) Clinical characteristics of 3,062 COVID-19 patients: a meta-analysis. J Med Virol 92:1902-1914. https://doi.org/10.1002/jmv.25884

22. Long H, Nie L, Xiang X, Li H, Zhang X, Fu X, Ren H, Liu W, Wang Q, Wu Q (2020) D-dimer and prothrombin time are the significant indicators of severe COVID-19 and poor prognosis. Biomed Res Int 2020:6159720-6159710. https://doi.org/10.1155/ 2020/6159720

23. Wu C, Chen X, Cai Y, Xia J, Zhou X, Xu S, Huang H, Zhang L, Zhou X, Du C, Zhang Y, Song J, Wang S, Chao Y, Yang Z, Xu J, Zhou X, Chen D, Xiong W, Xu L, Zhou F, Jiang J, Bai C, Zheng J, Song Y (2020) Risk factors associated with acute respiratory distress syndrome and death in patients with coronavirus disease 2019 pneumonia in Wuhan, China. JAMA Intern Med 180(7):1-11. https://doi.org/10.1001/jamainternmed.2020.0994

24. Fu YQ, Sun YL, Lu SW, Yang Y, Wang Y, Xu F (2020) Impact of blood analysis and immune function on the prognosis of patients with COVID-19. medRxiv 2020.04.16.20067587. https://doi.org/ $10.1101 / 2020.04 .16 .20067587$

Publisher's note Springer Nature remains neutral with regard to jurisdictional claims in published maps and institutional affiliations. 